\title{
PRECURSORES DE LA ENSEÑANZA DEL URBANISMO EN CHILE Primeros cursos y su influencia en la visión de ciudad desde la Arquitectura.
}

\author{
Precursors of the teaching of Urbanism in Chile \\ First courses and their influence on the vision of the city from Architecture
}

\author{
Matas, Isabel; \\ Universidad Mayor \\ isamatas@yahoo.com
}

\section{RESUMEN}

La investigación plantea el estudio del cruce entre los inicios de la enseñanza del urbanismo en dos escuelas de arquitectura durante la primera mitad del siglo XX en Chile. Se propone, una primera aproximación al estudio del desarrollo arquitectónico realizado por sus egresados, en el contexto social, económico y cultural de las décadas posteriores, como forma de determinar la influencia de la enseñanza del urbanismo en la formalización de la arquitectura en la ciudad. Manuel De Solà-Morales, hace notar la relevancia de la enseñanza del urbanismo en las escuelas de arquitectura como parte de la educación troncal de la disciplina para formar arquitectos con visión urbana. Se vuelve fundamental entender al arquitecto como constructor de ciudad y comprender que la enseñanza del urbanismo es una parte integral de la enseñanza de la arquitectura.

Palabras clave: enseñanza urbanismo, escuelas arquitectura, proyectos urbanos.

Bloque temático: teoría e historia de la ciudad.

\begin{abstract}
The investigation raises the study of the cross between the beginnings of the teaching of urbanism in two architecture schools during the first half of the 20th century in Chile. It is also proposed, a first approach to the study of the architectural development carried out by its graduates, in the social, economic and cultural context in the following decades, as a way to determine the influence of urban planning education in the formalization of architecture in the city. Manuel De Solà-Morales, notes the relevance of the teaching of urbanism in architecture schools as part of the core education of the discipline to train architects with urban vision. It becomes essential to understand the architect as a city builder and understand that the teaching of urbanism is an integral part of the teaching of architecture.
\end{abstract}

Keywords: urbanism teaching, architecture schools, urban projects.

Topic: theory and history of the city. 


\section{Introducción}

Antes de iniciar la investigación sobre la enseñanza del urbanismo y su implicancia en la construcción de una visión de ciudad, se hace necesaria la comprensión de lo que pasa en Chile desde fines del siglo XIX y hasta mediados del siglo $\mathrm{XX}$, como periodo de cambios sociales, políticos $\mathrm{y}$, sobre todo, en las manifestaciones físicas que eso conlleva en la ciudad. Solo así se podrá entrever la complejidad cultural del ambiente universitario de la época, que transita de forma contradictoria desde la enseñanza clásica hacia la moderna.

\section{Contexto histórico y urbano de la ciudad de Santiago}

Ya a fines del siglo XIX Santiago se había convertido en el centro urbano más importante del país, comenzando un proceso de emigración desde el campo a la ciudad que se mantendría durante todo el siglo XX. Mientras el centro fundacional se modernizaba con servicios que ofrecían las grandes ciudades europeas, la periferia de la ciudad crecía con una clase obrera cada vez más numerosa que vivía de forma miserable. "La modernización significó la búsqueda de un modelo de desarrollo urbano que solucionara los problemas que aquejaban a las ciudades chilenas. Para ello se basaron en un estudio de la realidad desde un punto de vista pragmático y funcionalista, aunque también fueron tomados en cuenta los levantamientos planimétricos usando, por vez primera en Chile, la fotografía aérea y llevando a cabo minuciosas visitas a terreno que los primeros urbanistas combinaron con la docencia universitaria. ... el gobierno contrató a un conocido urbanista vienés, Karl Brunner, para que se trasladara al país y aportara un programa de renovación urbana, aprovechando sus dotes para reformar la Facultad de Arquitectura de la Universidad de Chile" (De Ramón, 2015: 128).
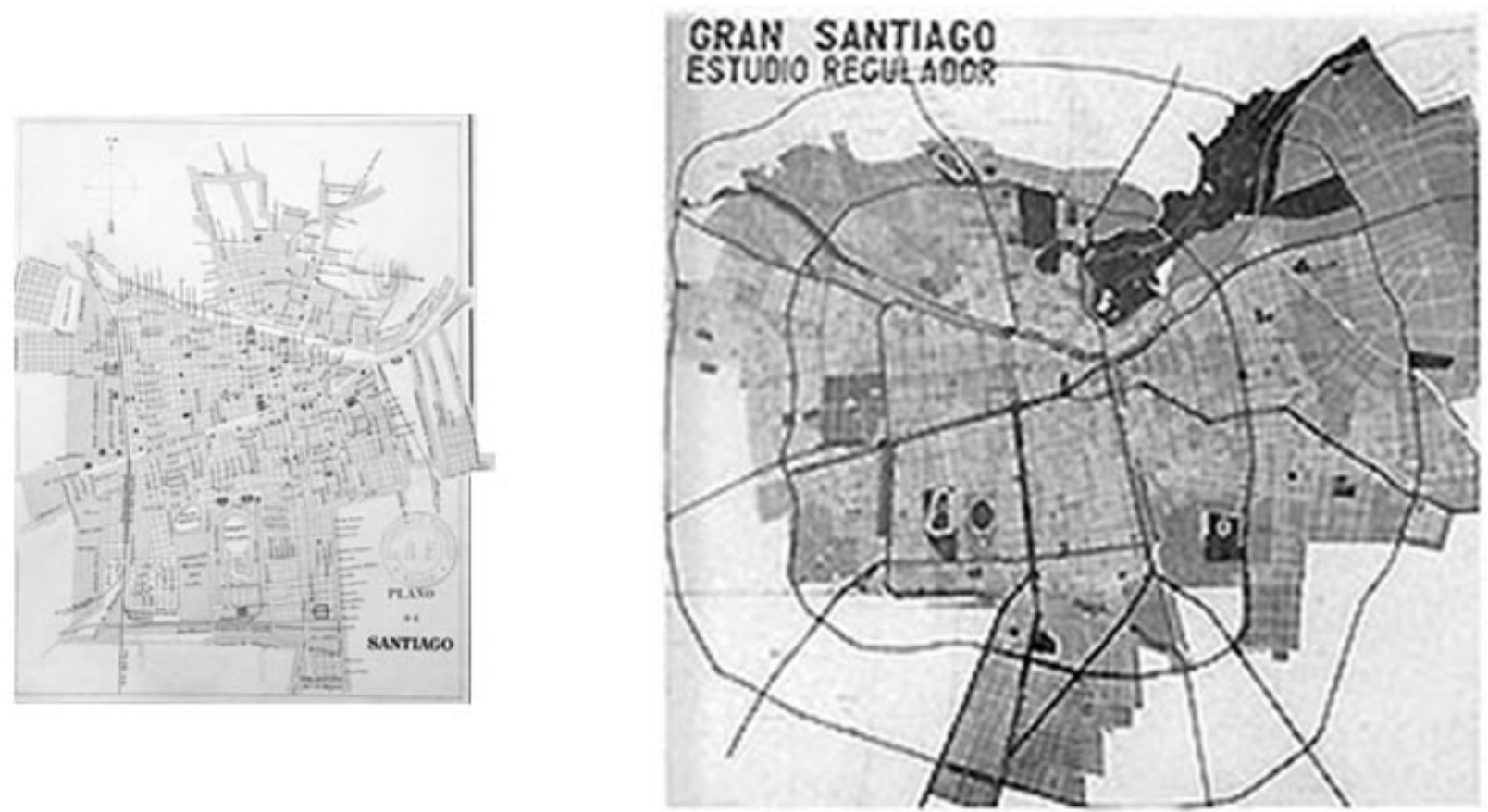

Fig. 01 Santiago 1887. Fuente: Archivo Visual de Santiago. Fig. 02 Santiago 1936, de L. Muñoz. Fuente: Pavez, 1996.

El salto del crecimiento urbano de Santiago es a partir de la década de 1930, producto en gran medida de la emigración resultante de la crisis del salitre, con la consiguiente necesidad de ordenamiento urbano de una ciudad que pasaba de 130.000 habitantes en 1875, a contar con casi 1.000 .000 hacia 1940. La ciudad cambia su escala (ver figuras 01 y 02, puestas a la misma escala), pasando de ser una pequeña ciudad de carácter doméstico a ser una metrópolis compleja que requiere de una planificación anticipada que organice y ordene un crecimiento urbano equilibrado y sostenible de acuerdo a un criterio pre establecido y unitario.

Para enfrentar este nuevo desafío de crecimiento, las autoridades de la época lo enfrentaron desde dos frentes: por un lado, en la consolidación de un cuerpo legal y normativo que delimitara las posibles acciones sobre la 
ciudad, y por otro a través de contrataciones de expertos urbanistas europeos, para proyectar la futura forma de la ciudad. Esto, como lo señala Gutiérrez (1996), conlleva aceptar o renegar las ideas preconcebidas que estos expertos exportan al país. Téllez (2009) afirma que al producirse la metropolización y la masificación de las ciudades chilenas, el crecimiento de las ciudades se da de forma dual, mientras la clase acomodada trata de parecerse a Europa, las clases más pobres malviven en condiciones casi indignas. Esa condición de insalubridad de la vivienda de la clase menos favorecida empieza a generar cierto ruido disciplinar y político a fines de los años treinta. Junto a eso, se comienzan a vislumbrar los primeros signos de la llegada de las ideas del movimiento moderno en Chile. Las universidades son sensibles a los movimientos de vanguardia. Algunos estudiantes y profesores de ambas escuelas de arquitectura que tuvieron la suerte de viajar y verlo en directo, y que al llegar de vuelta empiezan a denunciar fuertemente la enseñanza clásica que aún se dictaba en Chile, por medio de algunas revistas que empiezan a circular entre ciertos grupos de jóvenes vanguardistas.

\section{La enseñanza del Urbanismo en Chile}

La investigación se centra cronológicamente en las dos primeras escuelas que hubo en el país, la Universidad de Chile que comenzó a impartir cursos de arquitectura en 1849, y la Universidad Católica que comenzó en 1894. Interesa el periodo comprendido entre, la creación de los primeros cursos de urbanismo como parte integrante del plan de estudios de las dos escuelas de arquitectura, en 1928 en el caso de la Universidad de Chile y en 1929 en el caso de la Universidad Católica; y el periodo de transición entre la tradición histórica y la tradición moderna, que culmina en la refundación de la enseñanza de la arquitectura a partir de las ideas de la modernidad, en 1946 en la Universidad de Chile y 1949 en la Universidad Católica.

\subsection{Orígenes de la enseñanza de la Arquitectura en Chile}

La enseñanza de la arquitectura en Chile se inicia de manera informal a fines del siglo XVIII con clases particulares dadas por Joaquín Toesca a algunos pocos alumnos que llegaban a su casa, admirados por el trabajo que estaba haciendo en Santiago: la Casa de la Moneda y Los Tajamares del Mapocho. De esta manera, se da inicio a una forma de enseñanza que durará en Chile hasta mediados del siglo XIX, y que se explica en gran medida por la ausencia de una instrucción formal y sistematizada de la arquitectura y, por consiguiente, una falta de arquitectos nacionales. Esta, se vio reemplazada por invitaciones hechas por el Estado a arquitectos europeos para que vinieran a desarrollar encargos específicos al país, "Por encargo del Ministerio de Instrucción Pública, el representante de Chile en Francia contrataba en Paris a Claude Brunet de Baines" (Ballacey, 1984: 30). A esta decisión gubernamental se suma la petición del rector de la recién creada Universidad de Chile, primera Universidad del país fundada en 1842, Andrés Bello como parte del discurso inaugural de 1848 donde expone que "carecemos de arquitectos civiles, de ingenieros expertos en la construcción de caminos, puentes y toda clase de obras públicas. El Gobierno, ha aceptado con entusiasmo la idea de fundar en Santiago una escuela práctica de arquitectura civil, bajo la dirección de un ingeniero que debe llegar de Europa en un momento a otro" (Waisberg, 1999:31). Es así como la Universidad de Chile en 1849 y a través del recién llegado arquitecto Brunet de Baines crea la primera clase de arquitectura, que en su teoría dependía de la Facultad de Ciencias Físicas y Matemáticas.

La venida de Joaquín Toesca y la llegada de Brunet de Baines constituyen los dos principales hitos del comienzo de la enseñanza de la arquitectura en Chile, en cuyo ejercicio profesional posterior se vieron enfrentados en competencias aquellos que ejercían el oficio amparados en la experiencia de obra, con aquellos formados en las universidades "si Toesca introdujo en la cotidianidad la experiencia de la arquitectura hecha por arquitectos, a Brunet de Baines le correspondió introducir la docencia de la arquitectura, y un modo técnico de entenderla" (Aguirre, 2011:39). 


\subsubsection{Universidad de Chile}

Los primeros años de estudios transcurrieron de manera bastante precaria: con pocos alumnos, con la reorganización de la Facultad de Ciencias Físicas y Matemáticas, y finalmente con un periodo de receso que solo terminó con la llegada de un nuevo arquitecto de gobierno en 1857 desde Francia, Lucien Hénault (Waisberg, 1999). En 1858 con el traslado de la clase de arquitectura a la recién creada sección de Bellas Artes, se inician nuevamente las clases que esta vez tendrían una continuidad de ocho años, pero solamente dos arquitectos recibidos en el periodo. De esta manera, el curso de arquitectura se vuelve a cerrar por cinco años más y se reabre en 1872 bajo la dirección de Manuel Aldunate, chileno formado en Francia y que fue nombrado arquitecto de Gobierno el mismo año, quien para referirse a la situación plantea que "la profesión de arquitecto, que ha adquirido una importancia considerable entre nosotros, carecía en nuestra Universidad de los estudios más indispensables, por la prolongada ausencia del profesor del ramo, que había sido ocupado en otras comisiones. El Ministerio se hizo un deber se hacer cesar en el acto este grave mal. Hay gran escasez de arquitectos idóneos y hay por otra parte una gran conveniencia en ofrecer a la juventud del país la expectativa de una profesión honrosa y que ha llegado a ser muy lucrativa" (Waisberg, 1999:45). Al respecto cabe señalar que es mismo 1872 es el año en que se inicia la transformación urbana de Santiago propuesta por el intendente Benjamín Vicuña Mackenna, que evidentemente impulsa un auge en la edificación y en el interés por la profesión de arquitecto.

No es hasta 1896 que se logra ordenar y consolidar la enseñanza formal de la arquitectura en la Universidad de Chile. Esta enseñanza se imparte a través de un curso, otra vez dependiente de la Facultad de Ciencias Físicas y Matemáticas que recién vuelve a funcionar en 1899 con la contratación desde Francia de un nuevo arquitecto de Gobierno (Alegría, 1968; Waisberg, 1999). Con la llegada de Emilio Doyère al cargo destinado a organizar el curso de arquitectura, y con una nueva modificación al plan de estudios en 1903 que estipulaba la duración de la carrera en 5 años y el acceso anual de nuevos estudiantes (hasta 1894 el curso se iniciaba cada tres años), finalmente en el año 1907 los estudiantes matriculados fueron cincuenta y cinco (Anduaga, 1999).

Es interesante ver, primero, cómo pese al momento histórico y cultural que Chile enfrentaba en el cambio de siglo, la enseñanza de la arquitectura no logra establecerse de forma continua en 50 años (1849 -1899) a partir de la creación de los primeros cursos formales en la Universidad de Chile. Las razones son variadas, pero sin duda convergen la indefinición entre la visión anglosajona de la tradición politécnica o la francesa de la escuela de bellas artes, y a su vez, la indefinición de los límites conceptuales, teóricos y prácticos de lo que significa formar a un arquitecto y cuál es el rol del mismo en la sociedad.

Veinte años más tarde de la consolidación de la escuela de arquitectura, en 1928, se crea el primer curso de urbanismo y su primer profesor fue Alberto Schade quien arma un curso sobre higiene, redes urbanas, ornato y, en base a su experiencia personal a partir de su trabajo en el Departamento de Arquitectura de la Dirección de Obras Públicas desde 1904 a 1918 en que durante tres años permaneció como estudiante de arquitectura enviado por el gobierno a Francia. Al año siguiente, fue reemplazado como profesor por Karl Brunner, destacado ingeniero-arquitecto que fue invitado a Chile como asesor del Gobierno y profesor de la Universidad de Chile. Fue Rodolfo Oyarzún, el artífice de la invitación, ya que después de titularse de arquitecto en la misma escuela y habiendo hecho un curso de construcción en el Politécnico de Charlottenburgo en Berlín, estudió en la Academia de Bellas Artes de Viena durante dos años, donde tuvo la oportunidad de participar del Seminario de Urbanismo que dictaba Brunner desde 1924 (Pavez, 1992).

Así, en 1929 se dicta el curso de urbanismo con Karl Brunner de profesor en que se enseña el manejo racional y científico del desarrollo urbano como base de la preparación, y que tuvo un impacto inmediato tanto en la academia como en el ambiente de profesores que, a su vez estaba muy ligado al quehacer público. Brunner contó con la ayuda en la academia de Rodolfo Oyarzún y de Luis Muñoz, jefe de la sección de urbanismo de la Dirección de Obras Públicas como contraparte del Estado. 
A la partida de Brunner en 1932, Rodolfo Oyarzún se hace cargo del curso de urbanismo hasta 1946, creando además ese año un taller de urbanismo. Entre 1946 y 1952 es Luis Muñoz quien se desempeña como profesor de urbanismo en la escuela de arquitectura añadiéndole al curso algo del contenido de las teorías de la planificación regional (Pavez, 1992, 2016). A partir de 1963 ingresa como docente de urbanismo su ayudante, Juan Parrochia, quien después de titularse en 1953 estudia en Bélgica con Bardet, y a su vuelta a Chile acompaña la docencia con su trabajo en el Departamento de Urbanismo del Ministerio de Obras Públicas. En 1952 Juan Honold y Pastor Correa se titulan con la tesis llamada "Ensayo de Planificación del Gran Santiago", que resume la visión analítica y racional entregada por la escuela de la Universidad de Chile, y se convierten en la muestra evidente de cómo la visión académica de lo aprendido en la escuela puede llegar a convertirse en una visión de ciudad (ver figuras 04 y 05). Honold en una entrevista a Prieto (2007) reconoce la influencia inicial de CIAM y Bauhaus - se debe entender que vivió la crisis que llevó a la refundación de la escuela bajo los principios del movimiento moderno - y la influencia posterior de Geddes, Munford y Bardet.
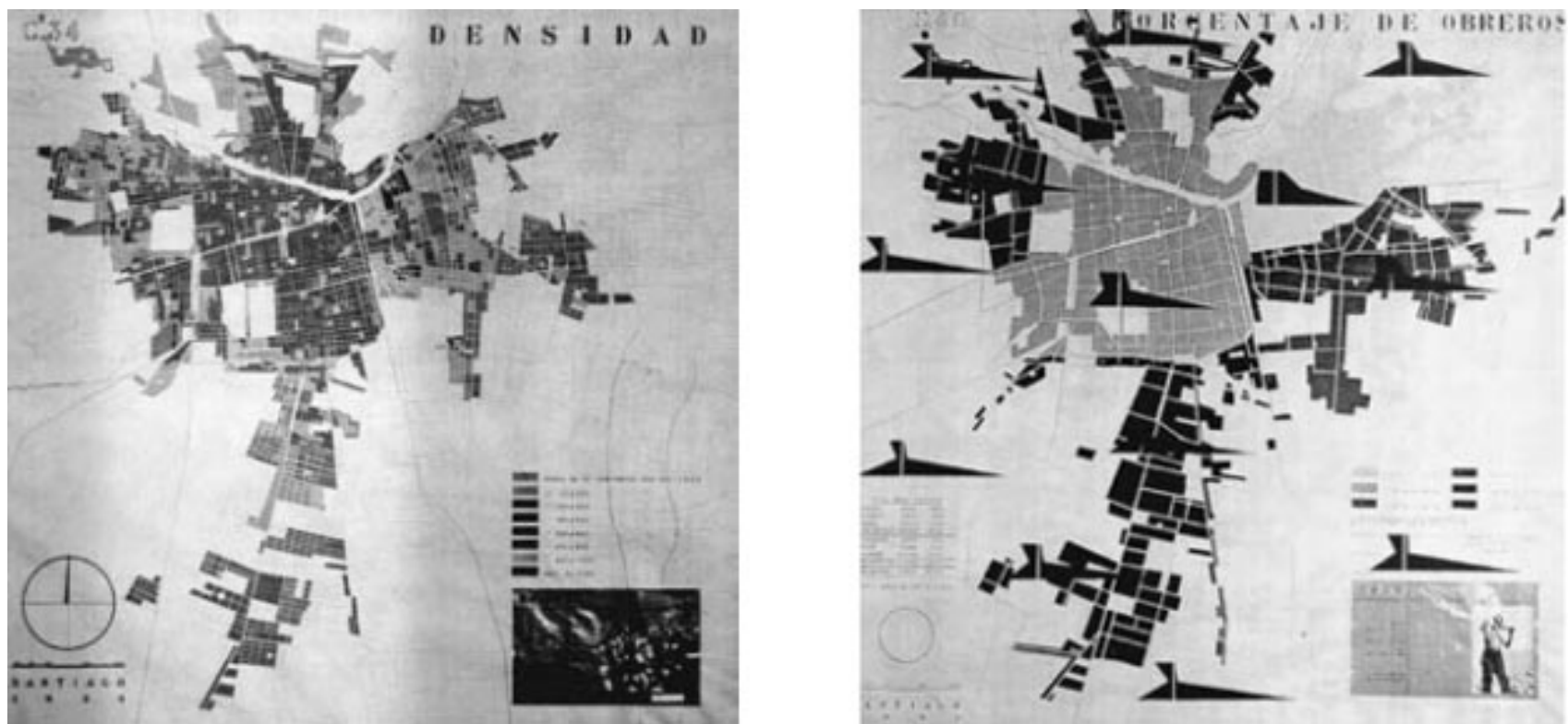

Fig. 04 y Fig. 05 Mapas de Santiago como análisis de la tesis de título de Correa y Honold, 1952. Fuente: Prieto, 2007.

Lo interesante de la Universidad de Chile es que desde el comienzo de la enseñanza del urbanismo se caracterizó por tener una relación directa entre la teoría y práctica del urbanismo, ya que sus profesores no se limitaron al ejercicio académico, sino que ejercieron su profesión -y por tanto sus influencias- desde el Estado, tomando las primeras decisiones de planeamiento de las ciudades en cargo públicos estratégicos.

\subsubsection{Universidad Católica}

Respecto a la Universidad Católica, en el año 1893 y cinco años después de la fundación de la Universidad, se abre por primera vez un curso de arquitectura. En 1894 se crea la Escuela de Arquitectura perteneciente a la Facultad de Ingeniería, bajo la dirección del presbítero Agustín Jara que desde un comienzo establece la enseñanza de arquitectura propiamente tal, como alternativa al curso que ya estaba funcionando de manera intermitente en la Universidad de Chile. Los primeros años se estudia en base a la Escuela de Matemáticas, incorporando cursos de dibujo y composición. En 1902 se modifica el plan de estudios con la llegada de Emilio Jecquier quien va aumentando poco a poco la influencia de los principios de la escuela de Bellas Artes.

En 1920 se crea la Facultad de Arquitectura y Bellas Artes, conformándose en la primera Facultad de Arquitectura del país, en parte como consecuencia del éxito obtenido por los trabajos enviados por parte de la escuela al Primer Congreso Panamericano de Arquitectos realizado en Montevideo. 
Los congresos se transformaron en decisivos en la consolidación de la enseñanza de la arquitectura en Chile, ya que crearon nuevas instancias de cultura arquitectónica que se tradujo en un aumento de profesionales en el mercado nacional. La publicación en 1924 de un álbum sobre los 30 primeros años de la Escuela de Arquitectura constituye "una prueba evidente de las estrechas relaciones existentes por entonces entre el mundo académico y el mundo profesional" (Pérez, 2017:34). El prestigio ganado por la Escuela de Arquitectura, sobre todo a nivel internacional, hace que sus titulados sientan orgullo de su enseñanza y se empiece a firmar como Arquitecto PUC y a su vez de tener el honor de formar parte de ella como docentes (Ballacey, 1984).

En 1929 aparece el primer curso de urbanismo, a cargo del ex alumno Alfredo Johnson, estructurado en base a los "fundamentos de la ciencia recientemente asimilada por él durante tres años de estudios en La Sorbonne" (Ballacey, 1984:116). Con este curso se marca el inicio formal de la enseñanza del urbanismo en la Universidad Católica, cuya "primera expresión es netamente formalista y se define como la posibilidad de establecer un juego estético a gran escala, constituyendo su campo de estudio los espacios más públicos de la ciudad: parques, plazas, conjuntos monumentales, etc. los que en un comienzo se estudiaban y analizaban a la luz de los principios de la composición clásica, con sus ejes, centros y diagonales, para luego, lentamente empezar a incorporar los principios de los innovadores Sitte, Howard, Le Corbusier y los CIAM" (Ballacey, 1984:117).
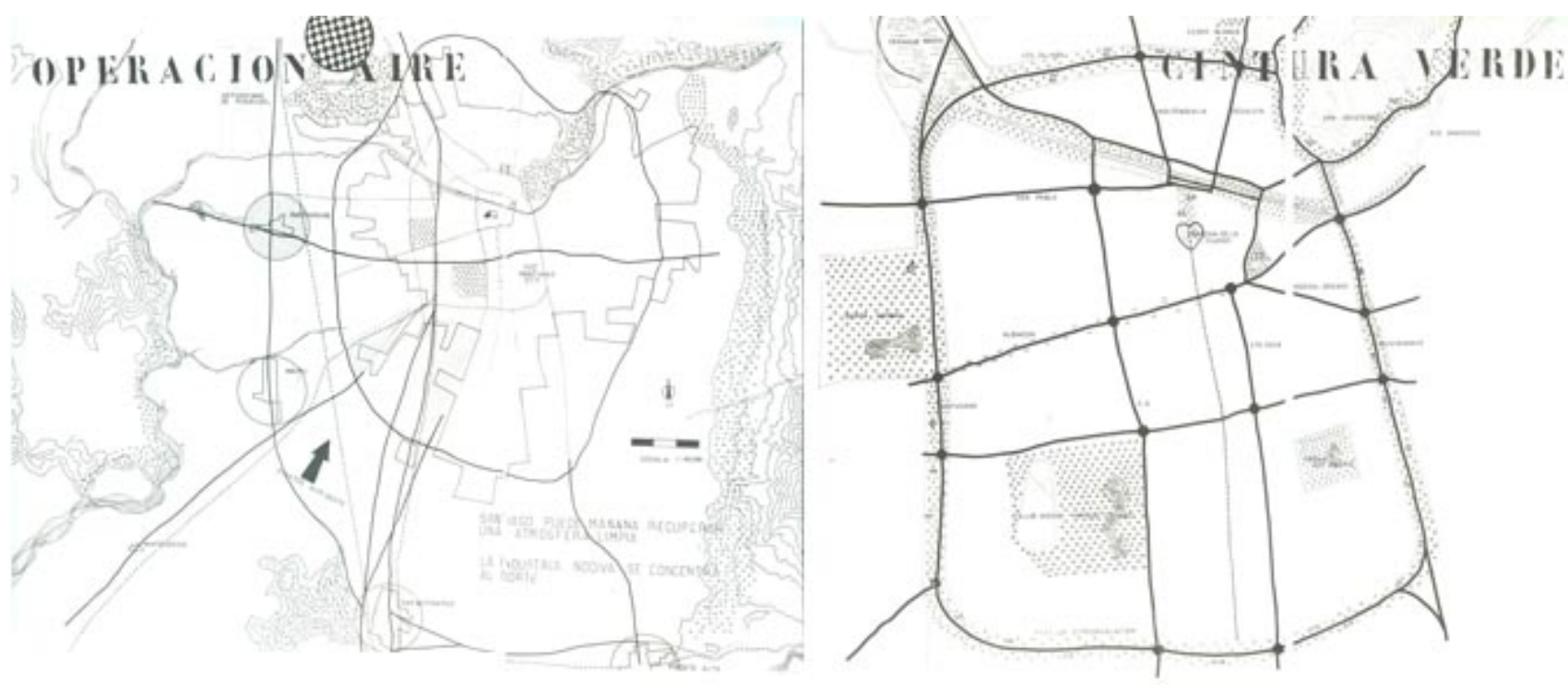

Fig. 06 Fig. 07 Mapas de Santiago como parte de la muestra del Seminario de Duhart de 1957. Fuente: Montealegre, 1994.

Se puede ver como los "estudios urbanísticos van adquiriendo un fuerte impulso y comienzan a constituirse en temas habituales para los proyectos finales" (Ballacey, 1984: 122), siendo el primero una "Base Pesquera" de Emilio Duhart en 1941. El mismo Duhart es profesor de taller de quinto año, y realiza con sus estudiantes en 1957 el "Seminario del Gran Santiago", patrocinado por la Universidad de Chile y el Ministerio de Obras Públicas (ver figuras 06 y 07). La exposición busca mostrar y hacer reflexionar sobre el futuro de la ciudad de Santiago, siendo la muestra de la Universidad Católica una de carácter urbana sin dejar de lado las propuestas arquitectónicas y su principal virtud está en "la globalidad de su visión" (Montealegre, 1994:77).

En un documento académico de 1957, se habla sobre los objetivos de la enseñanza del urbanismo en pre grado de arquitectura consistentes en "exponer claramente la función propia del arquitecto en el campo del urbanismo; preparando al alumno para que conozca otras disciplinas, estableciendo, no obstante, que los aspectos interdisciplinarios se deben despejar con claridad de las líneas que son indelegable y propias del arquitecto, las que son intransables. Se trata de reforzar el papel del arquitecto en cuanto tiene que ver con la construcción del espacio físico de la ciudad, y de enfocar las materias a ese propósito". Enfoque que muestra claramente cuál es la visión determinante de la visión urbana de la Universidad Católica históricamente. 


\section{Conclusiones del cruce académico de dos escuelas.}

Desde el inicio, y marcadas principalmente por la influencia de las corrientes europeas que vieron y estudiaron, ambas escuelas muestran una visión distinta en cuanto al rol del urbanismo en la enseñanza de la arquitectura. La Universidad de Chile desde sus inicios y hasta hoy tiene un marcado acento racional y técnico que puede verse desde sus cursos académicos hasta la obra de sus egresados, frente a la Universidad Católica que desde las bases de las Bellas Artes mantiene un marcado carácter compositivo fuertemente influido por la capacidad artística de su historia académica y de las obras de sus egresados. Hay un discurso frente al rector cuando el gobierno de Uruguay invita a la Universidad Católica al Congreso y Exposición Panamericana de Arquitectura en 1920, en que se envía a un buen número de trabajos de alumnos y titulados y, a una delegación de profesores en que "nuestra Escuela de Arquitectura en unión con la Escuela de Arquitectura de la Universidad de Chile, honraron a la Patria con el no superado éxito que obtuvieron sus esplendidas presentaciones" (Reyes. 1945: 6). Este documento es una muestra de la buena y cercana relación que había entre las dos escuelas de arquitectura. Pero, a su vez, hay un artículo del año 1936 escrito por un arquitecto de la Universidad Católica en que se refiere la Escuela de la Universidad de Chile como la "escuela rival, la escuela racionalista" con que se confirma una incipiente rivalidad a partir ¿quizá? de cómo se inicia la enseñanza del urbanismo en Chile. También Almandoz (2018) se refiere al urbanismo academista incipiente, e iniciado por Brunner, como uno de "carácter feudal a ojos de las nuevas generaciones más sensibles al urbanismo promulgado desde los CIAMs".

La diferencia de visión entre las dos escuelas de arquitectura viene dada desde un inicio, debido a las influencias teóricas de los profesores. Mientras en la Universidad de Chile los teóricos predominantes presentes en la enseñanza durante los años treinta y cuarenta fueron Bardet, Geddes y Munford; en la Universidad Católica fueron Sitté, Howard y los CIAM. Siguiendo un rápido resumen de las principales teorías enunciadas, y tal como lo indica Luque (2004) están por una parte Patrick Geddes (Escocia 1854 - Francia 1932) que establece el estudio minucioso del territorio como diagnóstico y reconocimiento de las características y tendencias de la región; Lewis Munford (USA 1895-1990) que también defiende la necesidad de un desarrollo regional, planteando que el problema de la ciudad no es una cuestión técnica, sino una cuestión cultural e histórica; y Gaston Bardet (Francia 1907-1989) que defiende el urbanismo social presentándose como continuador de las posturas academicistas que defendían la búsqueda de belleza en la ciudad. Por otra parte está Camille Sitté (Austria 1843-1903) que defiende la naturaleza artística de la ciudad y valora la ciudad existente; Ebenezer Howard (Inglaterra 1850-1928) que defiende la limitación del crecimiento de los núcleos urbanos y la mediación entre la ciudad y el campo; y los CIAM (Francia) en que bajo el alero de la Carta de Atenas defienden la relación racional entre los cuatro principios básicos, partiendo por la vivienda como máquina de habitar, el espacio de esparcimiento, de trabajo y de circulación. Se hace evidente entonces la visión predominante tanto en una como en otra escuela.

\section{Primeras aproximaciones de comparación de obras a partir de la visión de ciudad desde la enseñanza del urbanismo en las dos escuelas de arquitectura estudiadas.}

Interesa estudiar los inicios. Los inicios de la enseñanza de la arquitectura en Chile. Los inicios de la enseñanza del urbanismo dentro del pregrado de las dos primeras escuelas de arquitectura del país. Los inicios de la planificación territorial en Chile en cuanto a proyectos urbanos iniciales en los que se pueda corroborar la influencia de la visión de ciudad que transmite la enseñanza de urbanismo de las dos primeras escuelas de arquitectura. Los inicios, o no, del reflejo de esa enseñanza en la obra física: en la ciudad.

Como primera aproximación se quiere estudiar el Plan Regulador Intercomunal de Santiago y el Plan Regulador de Concepción, ambos de 1960. Y ambos como los primeros dos instrumentos de planificación territorial a nivel nacional que buscan ordenar y sistematizar el crecimiento de las dos ciudades más grandes de ese momento. 
Según Censo de 1960, Santiago tiene 1.907.378 habitantes y Concepción con 148.078 habitantes, siendo la tercera ciudad más habitada después de Valparaíso

El de Santiago es elaborado por ex alumnos de la Universidad de Chile, y el de Concepción por ex alumnos de la Universidad Católica. Interesa aproximarse a la comprensión de si efectivamente la visión de ciudad enseñada en urbanismo durante la formación universitaria, tiene repercusiones en el diseño final profesional.

\subsection{Plan Regulador Intercomunal de Santiago}

El diagnóstico inicia en el ámbito académico con la tesis de título de los arquitectos de la Universidad de Chile Pastor Correa y Juan Honold, quienes en 1952 desarrollan juntos el "Ensayo de Planificación del Gran Santiago". Después de la distinción máxima, es expuesto en la Universidad de Chile y genera un gran impacto en la opinión pública y universitaria (Vicuña, 2014). Santiago no tenía un plan desde los realizados por Brunner en la década del 30, esto, sumado a la necesidad de ordenar el crecimiento de la periferia, hace que el Ministerio de Obras Públicas contrate a Honold para el recién creado Departamento de Planeamiento.
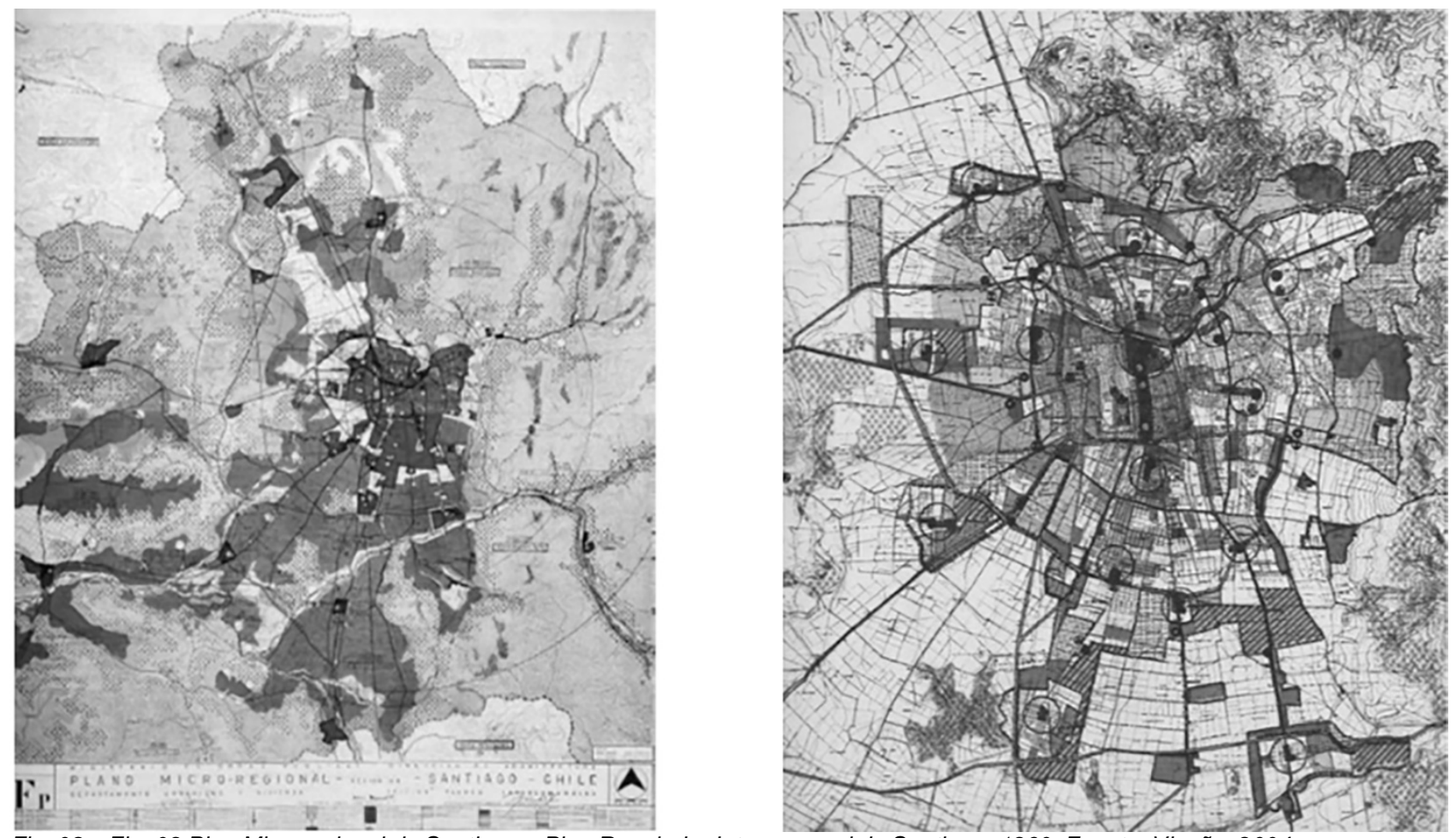

Fig. 08 y Fig. 09 Plan Microregional de Santiago y Plan Regulador Intercomunal de Santiago, 1960. Fuente: Vicuña, 2004.

El plan, y por consiguiente su manifestación física, está basado sobre la premisa que la ciudad debía ajustarse a la imagen pensada por los planificadores. Poduje (2006) analiza como la imagen del plan se materializa a través de tres políticas fundamentales: un límite urbano intercomunal, la zonificación de actividades y la construcción de una red vial estructurante.

\subsection{Plan Regulador de Concepción}

En cuanto al Plan Regulador de Concepción se inicia con un concurso en 1958 que gana el arquitecto de la Universidad Católica Emilio Duhart, quien se asocia con Roberto Goycoolea quien traslada su residencia a al sur para poder llevar a cabo esta obra (que se hace al mismo tiempo que la Universidad de Concepción). El proceso proyectual se inicia con un análisis del estado de la ciudad y un diagnóstico de las problemáticas y de las oportunidades con que se planifica a través de varias escalas de aproximación. El mismo Goycoolea (2004) 
señala que, al terminar el exhaustivo expediente urbano y el anteproyecto del plan, las principales intenciones del proyecto eran potenciar las características regionales e incorporar a la ciudad su geografía, y la densificación urbana para el centro de la ciudad para evitar la expansión horizontal. "La proposición más trascendente del plan regulador, que debiera rescatarse, incorporaba la ribera a la ciudad y consultaba un nuevo capitolio y centro administrativo en una isla artificial frente a ella" (Montealegre. 1994:83). El interior de la ciudad se organiza a través de una propuesta de vialidad estructurante y barrios como unidades vecinales en donde internamente se ordena mediante una zonificación. Además, se establece una clara zonificación que define tipos de usos de suelo con una densidad estimada, en donde en la articulación de las áreas verdes nuevamente va a los principios declarados en la Carta de Atenas para ordenarlos según temporalidad.
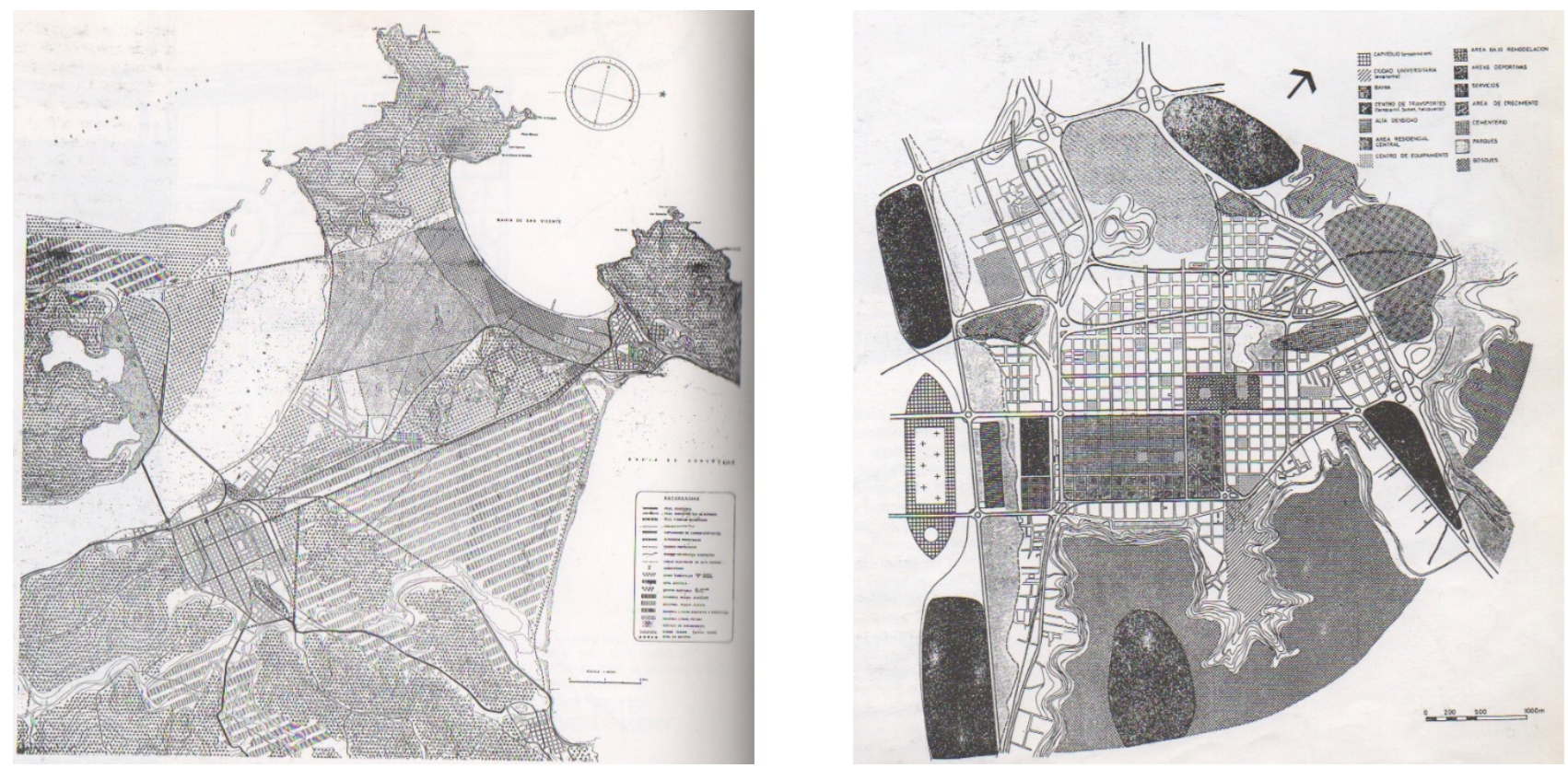

Fig. 10 y Fig. 11 Plan Regulador de Concepción, 1960. Fuente: Montealegre, 1994.

"El plan consideraba disposiciones y mejoras... importantes para el desarrollo de la ciudad. Algunas se mantienen en la planificación actual, otras han sufrido los cambios que podrían esperarse después de una sucesión de diferentes administraciones" (Montealegre. 1994:82).

\subsection{Primeras aproximaciones como conclusiones}

Habiendo hecho una primera comparación de ambos planes reguladores, y a la espera de hacer una matriz que pueda comparar cada tema por separado, se puede establecer como primer punto de conclusión que tanto la enseñanza del urbanismo en las escuelas de arquitectura estudiadas como el trabajo profesional de práctica urbana estudiada, se debaten entre las dos teorías mundiales imperantes de la época. Por un lado, las teorías científicas de los racionalistas del análisis urbano influenciados por Geddes y Munford, y por otro lado las teorías procedentes de la arquitectura moderna que determina la separación de funciones de la ciudad en habitar, trabajar y circular, influenciados por Le Corbusier. Pero, aunque apuntan a abordar las mismas áreas de conocimientos y los mismos objetivos generales, las perspectivas de análisis de la visión de ciudad de cada escuela de arquitectura son diferente a la hora de analizar los diseños generados por sus egresados.

En cuanto al Plan Regulador Intercomunal de Santiago la visión es de urbanismo racionalista científico, incorporando el componente social a través de las teorías de Bardet que son bastante transversales a ambos proyectos. En el caso del Plan Regulador de Concepción hay una visión algo utópica, heredada de la visión urbana del movimiento moderno, y aunque el plan mezcla ambas visiones incorporando ciertos principios científicos, finalmente es una visión predominantemente moderna. 


\section{BIBLIOGRAFÍA}

AA.VV. (1999). 150 años de enseñanza de la arquitectura en la Universidad de Chile: 1849-1999. Santiago: Facultad de Arquitectura y Urbanismo de la Universidad de Chile.

AGUIRRE, M. (2011). La arquitectura moderna en Chile. Santiago: Editorial Universitaria.

ALEGRÍA, R. (1968). Sobre la enseñanza de la arquitectura en la Universidad de Chile. Santiago: Escuela de Arquitectura de la Universidad de Chile, 1968.

ALMANDOZ, A., IBARRA, M. (2018). Vísperas del urbanismo en Latinoamérica, 1870-1930. Imaginarios, pioneros y disciplinas. Santiago: RIL editores - IEU UC.

BALLACEY, D., MÉNDEZ, R. (1984). Escuela de Arquitectura UC: 90 años de Escuela. Tomo 1. Santiago: Pontificia Universidad Católica de Chile.

BEDRACK, M. (1986). Urbanismo en Chile 1928 -1973. Revista CA № 46 p. 31-33.

BRUNNER, K. (1932). Santiago, su estado actual y futura formación. Santiago: Imprenta La Tracción.

DE RAMÓN, A. (2015). Santiago de Chile. Historia de una sociedad urbana. Tercera Edición. Santiago: Catalonia.

DE SOLÀ MORALES, M. (2008). Diez lecciones sobre Barcelona. Barcelona: Actar.

GOYCOOLEA, R. (2004). Patrimonio moderno y proyecto de ciudad: Plan Regulador de Concepción, de Emilio Duhart. Revista Urbano №10. Universidad del Bio-Bio.

GUTIÉRREZ, R. (1996). Modelo e imaginarios europeos en urbanismo americano. De Arquitectura 8, 2-3.

HOFER, A. (2003). Karl Brunner y el urbanismo europeo en América Latina. Bogotá: La Candelaria.

LUQUE, J.(coord..). (2004) Constructores de la ciudad contemporánea. Aproximación disciplinar a través de los textos. España: Departamento de Urbanismo de la E.T.S. de Arquitectura de la Universidad de Navarra.

MONTEALEGRE, A. (1994). Emilio Duhart arquitecto. Santiago: ARQ.

PAVEZ, M. (1992). Precursores de la enseñanza del Urbanismo en Chile: periodo 1928-1953. Revista de Arquitectura 3. Santiago: Facultad de Arquitectura y Urbanismo, Universidad de Chile. - (2016). La institución del Urbanismo en la Facultad de Arquitectura y Urbanismo de la Universidad de Chile. Tempranos aportes a la producción interdisciplinaria en investigación y docencia. Santiago: Universidad de Chile.

PÉREZ, F. (2016). Arquitectura en el Chile del siglo XX: Iniciando el nuevo siglo 1890-1930. Santiago: ARQ. (2017). Arquitectura en el Chile del siglo XX: Modernización y Vanguardia 1930-1950. Santiago: ARQ.

PRIETO, M. (2007). Juan Honold y Pastor Correa. Dos Urbanistas del Siglo XX. Santiago: LOM Ediciones.

REYES, T. (1945). Recuento de los primeros años del curso de arquitectura de la Universidad Católica de Chile y su desarrollo ulterior. Texto de un discurso pronunciado en la Facultad de arquitectura en 1945. Fondo Documental Escuela de Arquitectura. Archivo Biblioteca Facultad de Arquitectura.

VICUÑA, M.; HIDALGO, R. (2014). Premio Nacional de Urbanismo. 1971 - 2014. Santiago: MINVU.

WAISBERG, M. (1999). Creación y primera etapa: 1849-1899. AA.VV. 150 años de enseñanza de la arquitectura en la Universidad de Chile: 1849-1999. Santiago: FAU Universidad de Chile. 